\title{
Simple Moment Analysis for a Kinetic Study of the Chromatographic Behavior of Spherical Particles and Silica Monoliths
}

\author{
Kanji MiYabe \\ Department of Chemistry, Faculty of Science, Rikkyo University, 3341 Nishilkebukuro, Toshima, Tokyo 171-8501, \\ Japan
}

\begin{abstract}
A simple procedure of moment analysis was proposed for a kinetic study of the rate processes in the columns packed with full-porous spherical particles and silica monoliths. Previous chromatographic data measured in reversed-phase HPLC systems using Mightysil and Chromolith columns were analyzed by a simple moment analysis. The surface of the packing materials is chemically modified with octadecyl alkyl ligands. A mixture of methanol and water (80/20, v/v) and alkylbenzene homologous series $\left(\mathrm{C}_{6} \mathrm{H}_{5} \mathrm{C}_{\mathrm{n}} \mathrm{H}_{2 \mathrm{n}+1}, n=0-7\right)$ were used as the mobile-phase solvent and sample probes, respectively. More detailed information about the experimental conditions is provided in Supporting Information. The values of the intra-stationary phase diffusivity $\left(D_{\mathrm{e}}\right)$ and the surface diffusion coefficient $\left(D_{\mathrm{s}}\right)$, derived by the simple moment analysis, were almost the same as those by the conventional moment analysis. The simple moment analysis is effective for quantitative studies of mass transfer in chromatographic systems. The previous chromatographic data were also analyzed by assuming external porosity $\left(\varepsilon_{\mathrm{e}}\right)$ as typical values, i.e., 0.40 for spherical particles and 0.70 for silica monoliths. The resulting values of $D_{\mathrm{e}}$ and $D_{\mathrm{s}}$ were of the same order of magnitude as those derived by using $\varepsilon_{\mathrm{e}}$ experimentally measured. Even if $\varepsilon_{\mathrm{e}}$ is assumed to be typical values, the simple moment analysis is effective for preliminary studies of the mass-transfer kinetics in the columns.
\end{abstract}

Keywords Moment analysis, first absolute moment, second central moment, rate theory, mass transfer kinetics, intrastationary phase diffusivity, surface diffusion coefficient, spherical particles, silica monolith, column external porosity

(Received September 4, 2020; Accepted September 30, 2020; Advance Publication Released Online by J-STAGE October 9, 2020)

\section{Introduction}

High-performance liquid chromatography (HPLC) has been extensively used as one of the most powerful separation techniques. Various types of separation media have been used to achieve a faster separation with a high efficiency, such as silica monoliths and core-shell spherical particles. ${ }^{1,2}$ Compared with conventional full-porous spherical particles, the separation media have extremely different structural characteristics concerning the shapes and porosities. The chromatographic behavior of separation media has usually been analyzed from kinetic points of view by using ordinary rate equations, ${ }^{3,4}$ such as the van Deemter equation. However, it seems that the rate equations are not necessarily sufficient for a detailed and quantitative kinetic study of their chromatographic behavior because of the following three problems.

At first, the rate equations were not developed by considering the intrinsic structural characteristics of the separation media. Second, most of the rate equations neglect the existence of some mass-transfer processes in a column, i.e., external mass transfer and surface diffusion, ${ }^{5,6}$ which have an important influence on the column efficiency. Finally, the rate equations contain some fitting parameters, of which the physical definition is not necessarily clear. It is impossible to quantitatively derive kinetic

† To whom correspondence should be addressed.

E-mail: kmiyabe@rikkyo.ac.jp information about chromatographic separations, such as diffusivities and mass transfer coefficients, by analyzing the flow rate dependence of $\operatorname{HETP}(H)$.

In spite of the drawbacks of the ordinary rate equations, they have been extensively used because their description is usually quite simple. For example, the van Deemter equation is written as follows.

$$
H=A+\frac{B}{u}+C u
$$

where $u$ is the flow velocity of the mobile phase. As is well known, $A, B$, and $C$ are the coefficients of the A-, B-, and C-term, respectively. It is possible to divide the contributions of the A-, B-, and C-term to band broadening and to derive the values of $A, B$, and $C$. However, it is impossible to quantitatively determine the values of the diffusivities and mass transfer coefficients from those of the coefficients and to analyze chromatographic behavior in detail, even if the correlation between $H$ and $u$ is experimentally measured as accurately as possible.

Moment theory based on the general rate model of chromatography is one of the strategies for representing chromatographic behavior. It has been used for deducing information about the retention equilibrium and the masstransfer and reaction kinetics in the column from the first absolute $\left(\mu_{1 \mathrm{~A}}\right)$ and second central $\left(\mu_{2 \mathrm{C}}\right)$ moment of elution peaks. $^{7-10}$ In the moment theory, chromatographic processes in 
a column are described in detail by considering the contributions of plural rate processes to $\mu_{2 \mathrm{C}}$. Some of them are not considered in the conventional rate equations. The physical meaning of all parameters included in the moment equations is clearly defined. A framework of the moment equations for various separation media, which have different shapes (spherical particle, cylindrical fiber, and flat plate) and different porosities (fullporous, partially-porous, and non-porous), has already been developed while appropriately considering their structural characteristics. ${ }^{10-15}$

The moment theory is more effective than the rate theory for quantitatively and accurately analyzing the chromatographic behavior from kinetic points of view. However, it has not widely been used for analyzing the HPLC behavior. It seems to be one of the reasons that the moment analysis procedure of chromatographic data is mathematically more complicated. ${ }^{16-19}$ It is probably required to develop a convenient procedure for a moment analysis of the HPLC behavior by introducing some appropriate suppositions and simplifications.

In this study, at first, a simple procedure was considered for a moment analysis of the chromatographic behavior of two types of separation media, i.e., spherical particles and silica monoliths. Then, some previous chromatographic data were analyzed by applying the simple moment analysis procedure. The simple procedure seems to be effective for the spread of the moment analysis because it is easy for many researchers.

\section{Theory}

Moment equations for full-porous and non-porous separation media Moment equations of $\mu_{1 \mathrm{~A}}$ and $\mu_{2 \mathrm{C}}$ for full-porous and nonporous separation media of various shapes are represented as follows. ${ }^{10-12,14}$

$$
\begin{aligned}
\mu_{1 \mathrm{~A}}= & \frac{Z}{u}\left[1+\frac{\left(1-\varepsilon_{\mathrm{e}}\right)}{\varepsilon_{\mathrm{e}}}\left\{\varepsilon_{\mathrm{i}}+\left(1-\varepsilon_{\mathrm{i}}\right) K_{\mathrm{a}}\right\}\right] \\
\mu_{2 \mathrm{C}}= & \frac{Z}{u}\left[\frac{2 D_{\mathrm{L}}}{u^{2}}\left[1+\frac{\left(1-\varepsilon_{\mathrm{e}}\right)}{\varepsilon_{\mathrm{e}}}\left\{\varepsilon_{\mathrm{i}}+\left(1-\varepsilon_{\mathrm{i}}\right) K_{\mathrm{a}}\right\}\right]^{2}\right. \\
+ & \frac{2\left(1-\varepsilon_{\mathrm{e}}\right)}{\varepsilon_{\mathrm{e}}}\left[\left(1-\varepsilon_{i}\right) \frac{K_{\mathrm{a}}{ }^{2}}{k_{\mathrm{rk}}}+\left\{\frac{\xi^{2}}{\omega D_{\mathrm{e}}}+\frac{\xi}{\theta k_{\mathrm{f}}}\right\}\right. \\
& \left.\left.\left\{\varepsilon_{\mathrm{i}}+\left(1-\varepsilon_{\mathrm{i}}\right) K_{\mathrm{a}}\right\}^{2}\right]\right]
\end{aligned}
$$

where $Z$ is the column length, $u$ is the interstitial velocity of the mobile phase, $\varepsilon_{\mathrm{e}}$ is the void fraction of a column (external porosity), $\varepsilon_{\mathrm{i}}$ is the porosity of separation media (internal porosity), $K_{\mathrm{a}}$ is the retention equilibrium constant, $D_{\mathrm{L}}$ is the axial dispersion coefficient, $k_{\mathrm{rk}}$ is the rate constant of chemical interaction at the stationary phase surface, $k_{\mathrm{f}}$ is the external mass transfer coefficient, $D_{\mathrm{e}}$ is the effective intra-stationary phase diffusivity, $\theta$ and $\omega$ are respectively the numerical coefficients attached with $k_{\mathrm{f}}$ and $D_{\mathrm{e}}$, and $\xi$ is the diffusion distance, which corresponds to the radius of spherical particles and cylindrical fibers or to the thickness of flat plates. The value of $\varepsilon_{\mathrm{i}}$ for fullporous and non-porous separation media is in the range $0<\varepsilon_{\mathrm{i}}$ $<1$ and $\varepsilon_{\mathrm{i}}=0$, respectively. The value of $K_{\mathrm{a}}$ is equal to zero in the case of size exclusion chromatography. Because the influence of sample injection on $\mu_{1 \mathrm{~A}}$ and $\mu_{2 \mathrm{C}}$ is usually negligibly small, they are neglected in Eqs. (2) and (3).

At first, $\mu_{1 \mathrm{~A}}$ is represented as Eq. (2) irrespective of the shape of the separation media. On the other hand, the second central moment equation depends on their shapes. The basic formula for $\mu_{2 \mathrm{C}}$ is the same for all of the separation media. However, the values of $\theta$ and $\omega$ are systematically changed, i.e., 3 and 15 for spherical particles, 2 and 8 for cylindrical fibers, and 1 and 3 for flat plates. The values of $\theta$, i.e., 3, 2, and 1, probably represent the dimension of mass transfer in the stationary phases. The ratios of $\omega$ to $\theta$ are also systematically changed, i.e., 5, 4, and 3 . Giddings also reported the same values of $\omega$ for the three different shapes of packing materials, i.e., uniform film, cylindrical rod, and spherical bead. ${ }^{3}$ The numerical values of $\theta$ and $\omega$ reflect the geometrical difference between the packing materials. The moment equations for a full-porous cylindrical rod can be used for analyzing chromatographic data measured by using silica monolithic columns.

\section{Simplification of moment analysis procedure}

Equation (2) is the moment equation for $\mu_{1 \mathrm{~A}}$. Although it looks to be complicated, it is identical to the following equation.

$$
t_{\mathrm{R}}=t_{0}(1+k)
$$

where $t_{\mathrm{R}}$ is the retention time, $t_{0}$ is the elution time of an inert tracer, and $k$ is the retention factor. It was reported that $t_{\mathrm{R}}$ corresponded to $\mu_{1 \mathrm{~A}}$ with an error of less than $1 \%$, even if an elution peak showed asymmetrical profile, e.g., the asymmetry factor at $10 \%$ peak height is equal to $c a .2 .0 .^{20}$ In Eq. (2), it is required to derive $K_{\mathrm{a}}$ from $\mu_{1 \mathrm{~A}}$ using the values of $\varepsilon_{\mathrm{e}}$ and $\varepsilon_{\mathrm{i}}$. In contrast, Eq. (4) is quite simple and does not include $\varepsilon_{\mathrm{e}}$ and $\varepsilon_{\mathrm{i}}$. The retention behavior is represented by using only $k$, which is equal to the ratio of $\left(1-\varepsilon_{\mathrm{t}}\right) K_{\mathrm{a}}$ to $\varepsilon_{\mathrm{t}}$. The total porosity $\left(\varepsilon_{\mathrm{t}}\right)$ is equal to $\varepsilon_{\mathrm{e}}+\left(1-\varepsilon_{\mathrm{e}}\right) \varepsilon_{\mathrm{i}}$. In addition, $t_{0}$ is equal to $\varepsilon_{\mathrm{t}} Z /\left(\varepsilon_{\mathrm{e}} u\right)$. It is suggested that a different moment analysis procedure without using $K_{\mathrm{a}}$ may be more convenient for many researchers. The moment analysis procedure concerning $\mu_{2 \mathrm{C}}$ should also be simplified.

\section{Full-porous spherical particles}

A detailed explanation is provided in Supporting Information for the simplification of moment analysis procedure of chromatographic behavior in the column packed with fullporous spherical particles. The value of HETP $\left(H_{\text {total }}\right)$ is represented as follows.

$$
\begin{aligned}
H_{\text {total }}= & \frac{2 \varepsilon_{\mathrm{e}} \gamma_{1} D_{\mathrm{m}}}{u_{0}}+4 \gamma_{2} R_{\mathrm{p}}+\frac{2 R_{\mathrm{p}}{ }^{2} \lambda^{2}}{15\left(1-\varepsilon_{\mathrm{e}}\right) D_{\mathrm{e}}} u_{0} \\
& +\frac{2 R_{\mathrm{p}} \lambda^{2}}{3\left(1-\varepsilon_{\mathrm{e}}\right) \tau} u_{0}^{\frac{2}{3}}+\frac{2 v^{2}}{\left(1-\varepsilon_{\mathrm{e}}\right)\left(1-\varepsilon_{\mathrm{i}}\right) k_{\mathrm{rk}}} u_{0}
\end{aligned}
$$

where $u_{0}$ is the superficial velocity of the mobile phase $\left(=\varepsilon_{\mathrm{e}} u\right)$, $R_{\mathrm{p}}$ is the radius of spherical particles, $\gamma_{1}$ and $\gamma_{2}$ are the geometrical coefficients, and $D_{\mathrm{m}}$ is the molecular diffusivity. Equation (5) is simply written as follows by considering the flow rate dependence of $H_{\text {total }}$.

$$
H_{\text {total }}=\frac{B^{*}}{u_{0}}+A^{*}+C^{*} u_{0}+D^{*} u_{0}^{\frac{2}{3}}+E^{*} u_{0}
$$

In the case of reversed-phase liquid chromatography (RPLC), the contribution of the chemical interaction (e.g., adsorption/ desorption) kinetics, i.e., $\mathrm{E}^{*}$-term, to $H_{\text {total }}$ is usually assumed to be negligibly small because the reaction rate of physical adsorption is fast enough. ${ }^{8}$ Equation (6) is rearranged as follows. 


$$
H_{\text {total }}-D^{*} u_{0}^{\frac{2}{3}}=\frac{B^{*}}{u_{0}}+A^{*}+C^{*} u_{0}
$$

Equation (7) represents the correlation of $\left(H_{\text {total }}-D^{*} u_{0}^{2 / 3}\right)$ with $u_{0}$, and has the same formula with the van Deemter equation, i.e., Eq. (1). However, Eqs. (5) - (7) are essentially different from Eq. (1) in terms of the following two points. At first, the contribution of external mass transfer ( $\mathrm{D}^{*}$-term) to $H_{\text {total }}$ is considered in Eq. (7). Second, the definition and physical meanings of the parameter $C^{*}$ in Eq. (7) are clearly explained. As shown in Eqs. (5) - (7), $D_{\text {e }}$ can quantitatively be calculated from the value of $C^{*}$. The mechanism of mass transfer in the stationary phases can be analyzed in more detail by considering the contributions of pore diffusion and surface diffusion to $D_{\text {e }}$. These are obvious differences between the van Deemter equation and Eqs. (5) - (7).

\section{Silica monolith (full-porous cylindrical fiber)}

Similar to the case of full-porous spherical particles, simplification of the moment analysis procedure of the chromatographic behavior in a column packed with silica monoliths is explained in detail in Supporting Information. The original moment equations for silica monolithic columns, i.e., Eqs. (2) and (3) $(\theta=2, \omega=8)$, were developed by assuming as a first approximation that the unit structure of the continuous porous packing material in monolithic columns consisted of a cylindrical silica skeleton of the stationary phase including meso- and micropores, which were surrounded by a through macropore spaces filled with the mobile-phase solvent. ${ }^{10-12,14}$

Similar to Eq. (5), Eq. (8) is derived in the same manner for silica monoliths.

$$
\begin{aligned}
H_{\text {total }}= & \frac{2 \varepsilon_{\mathrm{e}} \gamma_{1} D_{m}}{u_{0}}+2 \varepsilon_{\mathrm{e}} \gamma_{3} R_{\mathrm{ss}}+\frac{2 R_{\mathrm{ss}}^{2} \lambda^{2}}{8\left(1-\varepsilon_{\mathrm{e}}\right) D_{\mathrm{e}}} u_{0} \\
& +\frac{2 R_{\mathrm{ss}} \lambda^{2}}{2\left(1-\varepsilon_{\mathrm{e}}\right) \varphi} u_{0}^{\frac{1}{2}}+\frac{2 v^{2}}{\left(1-\varepsilon_{\mathrm{e}}\right)\left(1-\varepsilon_{\mathrm{i}}\right) k_{\mathrm{rk}}} u_{0}
\end{aligned}
$$

where $R_{\mathrm{ss}}$ is the radius of the cylindrical stationary phase silica skeleton and $\gamma_{3}$ is the geometrical coefficient. Equation (8) is simply written as follows.

$$
H_{\text {total }}=\frac{B^{*}}{u_{0}}+A^{*}+C^{*} u_{0}+D^{*} u_{0}^{\frac{1}{2}}+E^{*} u_{0}
$$

Equation (9) is rearranged as follows.

$$
H_{\text {total }}-D^{*} u_{0}^{\frac{1}{2}}=\frac{B^{*}}{u_{0}}+A^{*}+C^{*} u_{0}
$$

The contribution of $\mathrm{E}^{*}$-term to $H_{\text {total }}$ is similarly assumed to be negligibly small in the case of RPLC systems. ${ }^{8}$ Equation (10) represents the correlation of $\left(H_{\text {total }}-D^{*} u_{0}^{1 / 2}\right)$ with $u_{0}$ and has the same formula with the van Deemter equation, i.e., Eq. (1).

\section{Experimental}

In a previous paper, ${ }^{21}$ the chromatographic behavior of two different types of separation media, i.e., full-porous spherical particle and silica monolith, was studied from kinetic points of view. The flow-rate dependence of $H_{\text {total }}$ was analyzed on the basis of the moment theory. In this study, the same experimental data were analyzed by applying a simple moment analysis
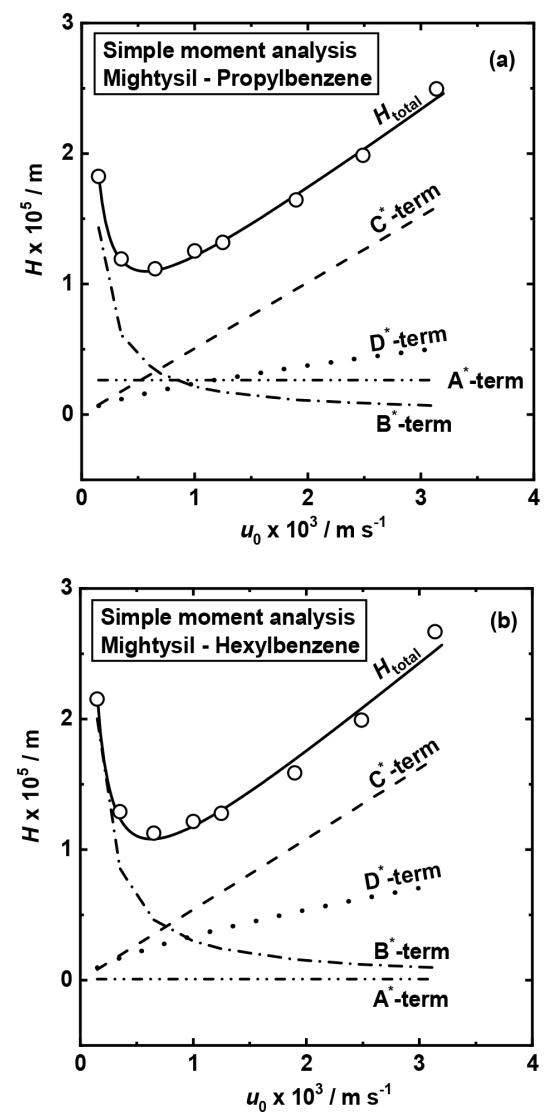

Fig. 1 Flow-rate dependence of $H$ and the contributions of $\mathrm{A}^{*_{-},} \mathrm{B} *_{-}$, $\mathrm{C}^{*}$-, and $\mathrm{D}^{*}$-term to $H$. Open circles represent the original experimental data of (a) propylbenzene and (b) hexylbenzene, which were measured by using the Mightysil column. The data were analyzed by a simple moment analysis procedure.

procedure. The basic information about HPLC experiments is briefly described in Supporting Information. Further details about the experiments and data analysis can be found in a previous paper. ${ }^{21}$

\section{Results and Discussion}

Full-porous spherical particles (Mightysil)

Figures $1 \mathrm{a}$ and $1 \mathrm{~b}$ illustrate the correlation between $H$ and $u_{0}$ for propylbenzene (PBz) and hexylbenzene (HBz) in the RPLC system using the Mightysil column, respectively. The solid line is the correlation line of open circles, which represent experimental data. Figure 1 also illustrates the flow rate dependence of the contributions of the $\mathrm{A}^{*_{-}}, \mathrm{B}^{*_{-}}, \mathrm{C}^{*_{-}}$, and $\mathrm{D}^{*_{-}}$ term to $H_{\text {total }}$. The chromatographic behavior of the Mightysil column was also analyzed by the conventional moment analysis. The profiles of the straight and curved lines obtained by the conventional moment analysis are superposed with those in Fig. 1 (not shown). Both the simple and conventional moment analyses provided the same results. At first, as explained from Eq. (S17) to Eq. (S20) in Supporting Information, the contribution of the D*-term to $H_{\text {total }}$ was calculated as Eq. (5) on the basis of the $k_{\mathrm{f}}$ value, which was estimated by using the Wilson-Geankoplis equation. Then, the flow-rate dependence of the right-hand side in Eq. (7) was analyzed to obtain the coefficients, $A^{*}, B^{*}$, and $C^{*}$. The values of the coefficients 
Table 1 Resulting values derived by the simple moment analysis

\begin{tabular}{|c|c|c|c|c|c|c|}
\hline Column & Solute & $k$ & $K_{\mathrm{a}}$ & $D_{\mathrm{e}} / \mathrm{m}^{2} \mathrm{~s}^{-1}$ & $D_{\mathrm{p}} / \mathrm{m}^{2} \mathrm{~s}^{-1}$ & $D_{\mathrm{s}} / \mathrm{m}^{2} \mathrm{~s}^{-1}$ \\
\hline \multirow[t]{4}{*}{ Mightysil } & \multirow[t]{2}{*}{ Propylbenzene } & 2.9 & 5.4 & $1.9 \times 10^{-10}$ & $3.2 \times 10^{-11}$ & $4.9 \times 10^{-11}$ \\
\hline & & $(2.9)$ & $(5.4)$ & $\left(1.9 \times 10^{-10}\right)$ & $\left(3.2 \times 10^{-11}\right)$ & $\left(5.2 \times 10^{-11}\right)$ \\
\hline & \multirow[t]{2}{*}{ Hexylbenzene } & 9.9 & 18 & $2.3 \times 10^{-10}$ & $2.6 \times 10^{-11}$ & $1.8 \times 10^{-11}$ \\
\hline & & $(9.9)$ & $(18)$ & $\left(2.3 \times 10^{-10}\right)$ & $\left(2.6 \times 10^{-11}\right)$ & $\left(1.9 \times 10^{-11}\right)$ \\
\hline \multirow[t]{4}{*}{ Chromolith } & \multirow[t]{2}{*}{ Propylbenzene } & 1.2 & 6.5 & $1.8 \times 10^{-10}$ & $5.1 \times 10^{-11}$ & $3.9 \times 10^{-11}$ \\
\hline & & $(1.2)$ & $(6.5)$ & $\left(1.7 \times 10^{-10}\right)$ & $\left(5.1 \times 10^{-11}\right)$ & $\left(3.6 \times 10^{-11}\right)$ \\
\hline & \multirow[t]{2}{*}{ Hexylbenzene } & 4.2 & 22 & $2.8 \times 10^{-10}$ & $4.1 \times 10^{-11}$ & $2.1 \times 10^{-11}$ \\
\hline & & $(4.2)$ & $(22)$ & $\left(2.7 \times 10^{-10}\right)$ & $\left(4.1 \times 10^{-11}\right)$ & $\left(2.0 \times 10^{-11}\right)$ \\
\hline
\end{tabular}

The values in parentheses are the retention and kinetic parameters derived by the conventional moment analysis.

were estimated by the least-squares method so that Eq. (7) represented the experimental curve profile between $H_{\text {total }}$ and $u_{0}$ as well as possible. In addition, the value of $C^{*}$ was analyzed to study the mass-transfer kinetics in the stationary-phase particles, as explained in the following.

As indicated in Eqs. (5) - (7), the values of $D_{\mathrm{e}}$ can be calculated from the slope of the straight lines for the $C^{*}$-term in Fig. 1. Table 1 lists the values of $D_{\mathrm{e}}$ determined by the simple moment analysis as $1.9 \times 10^{-10}$ and $2.3 \times 10^{-10} \mathrm{~m}^{2} \mathrm{~s}^{-1}$ for $\mathrm{PBz}$ and $\mathrm{HBz}$, respectively. The same values of $D_{\mathrm{e}}$ were also obtained by the conventional moment analysis. The mechanism of mass transfer in the stationary phase can be analyzed in more detail by considering the contributions of pore diffusion and surface diffusion to $D_{\mathrm{e}}$. As listed in Table 1, almost the same values of $D_{\mathrm{s}}$ were obtained by both the simple and conventional moment analyses. Table 1 also lists the values of pore diffusivity $\left(D_{\mathrm{p}}\right)$, which were estimated by Eqs. (S36) - (S38). The value of the tortuosity factor of internal pores $\left(k_{\mathrm{t}}^{2}\right)$ was calculated as 8.4 for the Mightysil column from the elution peak profiles of uracil. ${ }^{21}$ The comparison of $D_{\mathrm{e}}$ and $D_{\mathrm{p}}$ indicates that the contribution of surface diffusion to the molecular migration of $\mathrm{PBz}$ and $\mathrm{HBz}$ in Mightysil particles is $c a .5-8$ times larger than that of pore diffusion. It is indicated that surface diffusion has a predominant role for intraparticulate mass transfer. A determination procedure of $D_{\mathrm{p}}$ and $D_{\mathrm{s}}$ is explained in detail in Supporting Information.

The accuracy of the resulting values of diffusivities and mass transfer coefficients determined in this study is affected by the uncertainties in the estimate of some kinetic parameters, i.e., $D_{\mathrm{m}}, k_{\mathrm{f}}$, and $D_{\mathrm{p}}$, because literature correlations were used for estimating their values. At first, as indicated in Eq. (S37), the value of $D_{\mathrm{m}}$ was estimated by the Wilke-Chang equation. ${ }^{22}$ It is reported that the average error for estimating $D_{\mathrm{m}}$ by various correlations is slightly less than $10 \% .{ }^{23}$ The estimation error of $D_{\text {m }}$ affects the accuracy of the results in this study because the values of $k_{\mathrm{f}}$ and $D_{\mathrm{p}}$ were calculated on the basis of the estimated value of $D_{\mathrm{m}}$.

As explained by Eqs. (S17) - (S19) in Supporting Information, the $k_{\mathrm{f}}$ value was estimated by the Wilson-Geankoplis equation. The error of $k_{\mathrm{f}}$ made by the propagation of the uncertainty of $D_{\mathrm{m}}$ should be smaller than about $6 \%$ because $D_{\mathrm{m}}$ is estimated with an error of $10 \%$ or less and because $k_{\mathrm{f}}$ is proportional to $D_{\mathrm{m}}{ }^{2 / 3}$. Figures 1 and 2 indicate that the value of $D^{*}$-term increases with an increase in $u_{0}$ and that its contribution to $H_{\text {total }}$ is about $30 \%$ at maximum in the high flow rate range. It seems that the influence of the uncertainty of $k_{\mathrm{f}}$ on the error of the moment analysis is about $2 \%$ at maximum.

As described by Eq. (S36) in Supporting Information, the uncertainty of $D_{\mathrm{p}}$ is proportional to that of $D_{\mathrm{m}}$. However, the contribution of surface diffusion to intraparticulate mass transfer in Mightysil particles is $c a .5-8$ times larger than that of pore diffusion. This means that surface diffusion has a predominant role in the intraparticle diffusion as much as $c a .83-89 \%$. The influence of the error made in the estimation of $D_{\mathrm{p}}$ on the determination of $D_{\mathrm{s}}$ is calculated to be $c a .1 .1-1.7 \%$. Similarly, as described later, the contribution of surface diffusion to intrastationary phase diffusion is larger than that of pore diffusion by a factor of $c a .2 .5-6.0$ for Chromolith column. The error of $D_{\mathrm{s}}$ made by propagation of the uncertainty of $D_{\mathrm{m}}$ should be about $1.4-2.9 \%$ because about $71-86 \%$ of solute molecules migrate in silica-monolith by surface diffusion. The inaccuracy of $D_{\mathrm{p}}$ does not seriously affect the accuracy of $D_{\mathrm{s}}$. In addition, the information about other parameters, e.g., $K_{\mathrm{h}}$ and $k_{\mathrm{t}}^{2}$, are necessary when the values of $k_{\mathrm{f}}$ and $D_{\mathrm{p}}$ are estimated. The error in the estimations described above is responsible for the accuracy of the moment analysis. It seems that the overall error of the moment analysis is on the order of several percent.

As indicated in Eqs. (5) - (7), only $\varepsilon_{\mathrm{e}}$ is necessary as information about the porosities for the simple moment analysis when the $\mathrm{E}^{*}$-term is neglected. Various methods have been proposed for measuring $\varepsilon_{\mathrm{e}}$ of HPLC columns packed with porous materials, e.g., Donan exclusion method, ${ }^{24}$ inverse size exclusion chromatography, ${ }^{25}$ and total pore blocking. ${ }^{26}$ Simple moment analysis of the chromatographic behavior of HPLC columns packed with spherical particles can accurately be conducted by using $\varepsilon_{\mathrm{e}}$ experimentally measured by the methods. On the other hand, it is reported that $\varepsilon_{\mathrm{e}}$ is typically around $0.40-0.41$ in analytical columns packed with spherical particulate packing materials. ${ }^{9}$ Giddings also indicates that a fairly well-packed chromatographic column will typically have $\varepsilon_{\mathrm{e}}=0.4 \pm 0.03 .^{3} \quad$ Similar values of $\varepsilon_{\mathrm{e}}$ were experimentally measured in the range from $c a$. 0.35 to $0.43 .{ }^{27-32}$ It would be convenient that the simple moment analysis of chromatographic behavior of HPLC columns packed with spherical particles can be conducted by using the empirical $\varepsilon_{\mathrm{e}}$ values.

Figure S1a illustrates the correlation between $D_{\mathrm{e}}$ and $\varepsilon_{\mathrm{e}}$. The value of $\varepsilon_{\mathrm{e}}$ was hypothetically changed in the range from 0.35 to 0.45 , which was wider than the previous observations. ${ }^{3,9}$ The $D_{\text {e }}$ values were calculated at the different $\varepsilon_{\mathrm{e}}$ values by the simple moment analysis from the $H-u_{0}$ plots for $\mathrm{PBz}$ and $\mathrm{HBz}$ in Figs. 1a and $1 \mathrm{~b}$. When $\varepsilon_{\mathrm{e}}$ is assumed to be 0.40 , the values of $D_{\mathrm{e}}$ are $1.6 \%$ and $2.6 \%$ larger than those of $D_{\mathrm{e}}$ at $\varepsilon_{\mathrm{e}}=0.39$ for $\mathrm{PBz}$ and $\mathrm{HBz}$, respectively. Although the $\varepsilon_{\mathrm{e}}$ value experimentally measured was 0.39 in this case, the $D_{\mathrm{e}}$ values were derived with an error of a few percent when $\varepsilon_{\mathrm{e}}$ was assumed to be 0.40 . Figure $\mathrm{S} 1 \mathrm{a}$ indicates that the relative error of $D_{\mathrm{e}}$ at $\varepsilon_{\mathrm{e}}$ between 0.35 and 0.45 against $D_{\mathrm{e}}$ at $\varepsilon_{\mathrm{e}}=0.40$ is $7.1-9.2 \%$ and $12-16 \%$ for $\mathrm{PBz}$ and $\mathrm{HBz}$, respectively. It would be impossible to describe the correlation between $D_{\mathrm{e}}$ and $\varepsilon_{\mathrm{e}}$ by using a simple equation. As indicated in Eq. (S14), all of the contributions of 
$H_{\mathrm{ax}} *, H_{\mathrm{d}}{ }^{*}, H_{\mathrm{f}} *$, and $H_{\mathrm{rk}} *$ to $H_{\text {total }}$ depend on the value of $\varepsilon_{\mathrm{e}}$ because they contain $\varepsilon_{\mathrm{e}}$. In addition, as described in Eqs. (S8) and (S12), $\varepsilon_{\mathrm{e}}$ is also included in both the functions for $\lambda$ and $v$. As explained in Eqs. (S21) and (S22), the value of $D_{\mathrm{e}}$ was calculated from that of $C^{*}$. Although the dependence of $D_{\mathrm{e}}$ on $\varepsilon_{\mathrm{e}}$ is relatively complicated as explained above, the moment analysis is useful to clarify the correlation between $D_{\mathrm{e}}$ and $\varepsilon_{\mathrm{e}}$ as illustrated in Figs. S1a and S2a.

Figure $\mathrm{S} 1 \mathrm{~b}$ similarly illustrates the correlation between $D_{\mathrm{s}}$ and $\varepsilon_{\mathrm{e}}$. The value of $D_{\mathrm{s}}$ was calculated by Eqs. (S34) and (S36) (S38) from that of $D_{\mathrm{e}}$, which was obtained by a simple moment analysis at different $\varepsilon_{\mathrm{e}}$ values. When $\varepsilon_{\mathrm{e}}$ is assumed to be 0.40 , the values of $D_{\mathrm{s}}$ are $4.1 \%$ and $5.0 \%$ larger than those of $D_{\mathrm{s}}$ at $\varepsilon_{\mathrm{e}}=0.39$ (experimentally measured) for $\mathrm{PBz}$ and $\mathrm{HBz}$, respectively. It seems that the $D_{\mathrm{s}}$ values were derived with an error of several percent when $\varepsilon_{\mathrm{e}}$ was assumed to be 0.40 . Figure $\mathrm{S} 1 \mathrm{~b}$ indicates that the relative error of $D_{\mathrm{s}}$ at $\varepsilon_{\mathrm{e}}$ between 0.35 and 0.45 against $D_{\mathrm{s}}$ at $\varepsilon_{\mathrm{e}}=0.40$ is $c a .17-23 \%$ and $21-31 \%$ for $\mathrm{PBz}$ and $\mathrm{HBz}$, respectively. It is concluded from the results described above that preliminary information about the mass transfer in the stationary phase particles can be obtained with a moderate accuracy by assuming $\varepsilon_{\mathrm{e}}=0.40$. When accurate results of moment analysis are required, it is required to experimentally measure the value of $\varepsilon_{\mathrm{e}}$.

\section{Full-porous cylindrical fiber (Chromolith)}

Similar to Fig. 1, Fig. 2 illustrates the correlation between $H$ and $u_{0}$ for $\mathrm{PBz}$ and $\mathrm{HBz}$ in the RPLC system using the Chromolith column. Figure 2 also illustrates experimental data (open circles) and the contributions of the $\mathrm{A}^{*_{-}}, \mathrm{B}^{*_{-}}, \mathrm{C}^{*_{-}}$, and $\mathrm{D}^{*}$-term to $H_{\text {total }}$, which was analyzed by a simple moment analysis. The profiles of the straight and curved lines in Fig. 2 are superposed with those obtained by the conventional moment analysis (not shown). Both the simple and conventional moment analyses provided the same results for the chromatographic behavior of the Chromolith column.

As indicated in Eqs. (8) - (10), the values of $D_{\mathrm{e}}$ can be calculated from the slope of the straight lines for the $C^{*}$-term in Fig. 2. As listed in Table 1 , the value of $D_{\mathrm{e}}$ was calculated as $1.8 \times 10^{-10}$ and $2.8 \times 10^{-10} \mathrm{~m}^{2} \mathrm{~s}^{-1}$ for $\mathrm{PBz}$ and $\mathrm{HBz}$, respectively, by the simple moment analysis. On the other hand, $D_{\mathrm{e}}$ was respectively calculated as $1.7 \times 10^{-10}$ and $2.7 \times 10^{-10} \mathrm{~m}^{2} \mathrm{~s}^{-1}$ for $\mathrm{PBz}$ and $\mathrm{HBz}$ by the conventional moment analysis. Almost the same values of $D_{\mathrm{e}}$ were obtained by both the simple and conventional moment analyses.

Similar to the Mightysil column, it was tried to quantitatively analyze the mechanism of mass transfer in the stationary phase of the Chromolith column. The value of $D_{\mathrm{s}}$ was calculated in the same manner. The hindrance parameter of internal pores $\left(K_{\mathrm{h}}\right)$ was estimated by Eq. (S38) around 0.80 for the three solutes in the RPLC systems using the Chromolith column. It was reported for the Chromolith column that the value of $k_{\mathrm{t}}^{2}$ ranged from 5.5 to 6.7 .33 In this study, an average value, i.e., 6.1 , was used as $k_{\mathrm{t}}^{2}$, which was a typical value for porous silica gel. ${ }^{8}$ As listed in Table 1, almost the same values of $D_{\text {s }}$ were obtained by both the simple and conventional moment analyses. Table 1 also lists the values of $D_{\mathrm{p}}$, which were estimated by Eqs. (S36) - (S38). The values of $D_{\mathrm{e}}$ are several times larger than those of $D_{\mathrm{p}}$, suggesting that surface diffusion plays an important role for the molecular migration in the stationary phase of the Chromolith column. The contribution of surface diffusion to intra-stationary phase diffusion of $\mathrm{PBz}$ and $\mathrm{HBz}$ is larger than that of pore diffusion by a factor of $c a .2 .5-6.0$.

As indicated in Eqs. (8) - (10), only $\varepsilon_{\mathrm{e}}$ is necessary for the simple moment analysis when the $\mathrm{E}^{*}$-term is neglected. It has
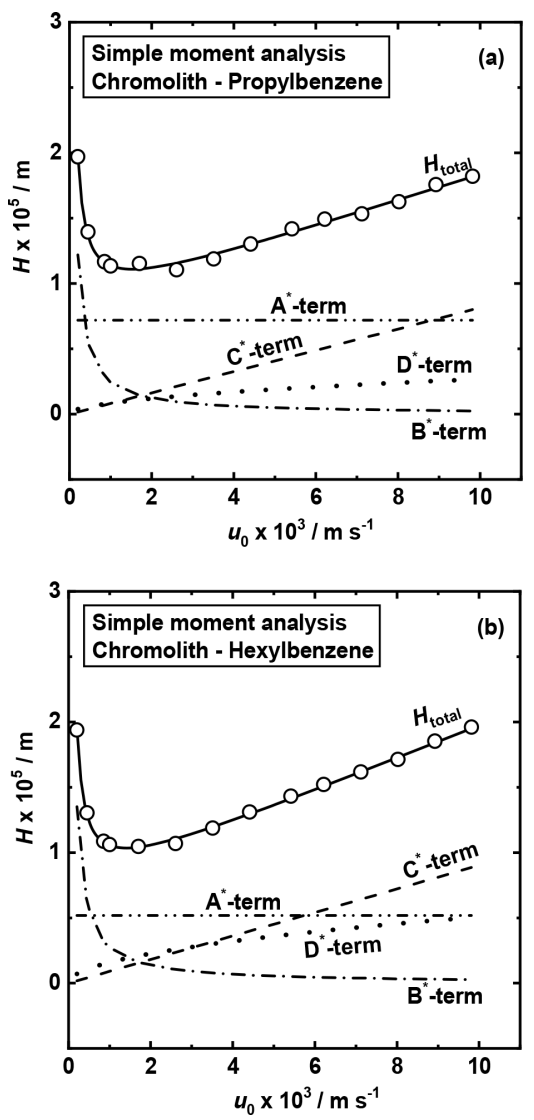

Fig. 2 Flow-rate dependence of $H$ and the contributions of $\mathrm{A}^{*_{-}}, \mathrm{B}^{*_{-}}$, $\mathrm{C}^{*}$-, and $\mathrm{D}^{*}$-term to $H$. The open circles represent the original experimental data of (a) propylbenzene and (b) hexylbenzene, which were measured by using a Chromolith column. The data were analyzed by a simple moment analysis procedure.

been reported that $\varepsilon_{\mathrm{e}}$ is usually ranging from 0.65 to 0.75 in Chromolith columns. ${ }^{27,29,34,35}$ The values of $\varepsilon_{\mathrm{e}}$ were experimentally measured in the same range. ${ }^{31,35,36}$ It has been tried to analyze the chromatographic behavior of the Chromolith column by using the empirical $\varepsilon_{\mathrm{e}}$ values. Figure S2a illustrates the correlation between $D_{\mathrm{e}}$ and $\varepsilon_{\mathrm{e}}$. The value of $\varepsilon_{\mathrm{e}}$ was hypothetically changed in the range of 0.65 to 0.75 . The $D_{\mathrm{e}}$ values were calculated at the different $\varepsilon_{\mathrm{e}}$ values by the simple moment analysis from the $H-u_{0}$ plots for PBz and $\mathrm{HBz}$ in Figs. 2a and 2b. When $\varepsilon_{\mathrm{e}}$ is assumed to be 0.70 , the values of $D_{\mathrm{e}}$ are $2.2 \%$ and $4.3 \%$ larger than those of $D_{\mathrm{e}}$ at $\varepsilon_{\mathrm{e}}=0.69$ for $\mathrm{PBz}$ and $\mathrm{HBz}$, respectively. Although the experimental value of $\varepsilon_{\mathrm{e}}$ was 0.69 , the $D_{\mathrm{e}}$ values were derived with an error of several percent when $\varepsilon_{\mathrm{e}}$ was assumed to be 0.70 . Figure $\mathrm{S} 2 \mathrm{a}$ indicates that the relative error of $D_{\mathrm{e}}$ at $\varepsilon_{\mathrm{e}}$ between 0.65 and 0.75 against $D_{\mathrm{e}}$ at $\varepsilon_{\mathrm{e}}=0.70$ is $9.7-15 \%$ and $17-29 \%$ for $\mathrm{PBz}$ and $\mathrm{HBz}$, respectively.

Figure $\mathrm{S} 2 \mathrm{~b}$ illustrates the correlation between $D_{\mathrm{s}}$ and $\varepsilon_{\mathrm{e}}$. The value of $D_{\mathrm{s}}$ was calculated from that of $D_{\mathrm{e}}$, which was obtained by the simple moment analysis at the different $\varepsilon_{\mathrm{e}}$ values. When $\varepsilon_{\mathrm{e}}$ is assumed to be 0.70 , the values of $D_{\mathrm{s}}$ are $1.3 \%$ and $2.4 \%$ larger than those of $D_{\mathrm{s}}$ at $\varepsilon_{\mathrm{e}}=0.69$ (experimentally measured) for $\mathrm{PBz}$ and $\mathrm{HBz}$, respectively. It seems that the $D_{\mathrm{s}}$ values were derived with an error of a few percent when $\varepsilon_{\mathrm{e}}$ was assumed to be 0.70 . Figure $\mathrm{S} 2 \mathrm{~b}$ indicates that the relative error of $D_{\mathrm{s}}$ at $\varepsilon_{\mathrm{e}}$ between 0.65 and 0.75 against $D_{\mathrm{s}}$ at $\varepsilon_{\mathrm{e}}=0.70$ is $c a$. $5.8-7.0 \%$ and $10-14 \%$ for $\mathrm{PBz}$ and $\mathrm{HBz}$, respectively. It is only required to measure $\varepsilon_{\mathrm{e}}$ experimentally when more accurate results of 
moment analysis are necessary.

\section{Conclusions}

It was tried to simplify the moment analysis procedure for a kinetic study on chromatographic behavior of spherical particles and silica monoliths. The simple procedure for a moment analysis of $\mu_{2 \mathrm{C}}$ was proposed without using $K_{\mathrm{a}}$ and $\varepsilon_{\mathrm{i}}$. However, similar to the conventional moment analysis, the contributions of axial molecular diffusion, Eddy diffusion, external mass transfer, intra-stationary phase diffusion, and reaction kinetics on the stationary phase surface to the variance of an elution peak could be quantitatively analyzed. Some chromatographic data measured by using the Mightysil column (full-porous spherical particles) and the Chromolith column (silica monoliths) were analyzed by applying the simple moment analysis procedure. Both the simple and conventional moment analysis procedures provided almost the same values of $D_{\mathrm{e}}$ and $D_{\mathrm{s}}$. The simple moment analysis is effective for quantitatively studying the mechanism of mass transfer in the stationary phases in detail with considering the contributions of pore diffusion and surface diffusion to $D_{\mathrm{e}}$. It was also tried to analyze the same chromatographic data by assuming $\varepsilon_{\mathrm{e}}=0.40$ for spherical particles and 0.70 for silica monoliths. Although the values of $D_{\mathrm{e}}$ and $D_{\mathrm{s}}$ change depending on the $\varepsilon_{\mathrm{e}}$ values, they were of the same order of magnitude with each other. Even if $\varepsilon_{\mathrm{e}}$ is hypothetically assumed to be typical values, the simple moment analysis is effective for a preliminary study of the mass-transfer kinetics in the columns. When more accurate results of moment analysis are required, the value of $\varepsilon_{\mathrm{e}}$ should experimentally be measured. In this study, the simple procedure of moment analysis was proposed for the analysis of chromatographic data measured by using HPLC columns packed with spherical particles and cylindrical rod fibers, such as silica monoliths. There is no necessary condition for a simple moment analysis, e.g., the types of solutes and stationary phases and the size and porosity of the packing materials, because it was theoretically developed on the basis of the general rate model of chromatography.

\section{Acknowledgements}

This work was supported by JSPS KAKENHI Grant Number $20 \mathrm{~K} 05570$.

\section{Supporting Information}

The simplification of moment analysis procedure is explained in detail. Supporting Information provides a detailed explanation about chromatographic experimental conditions and kinetic analysis of intraparticulate mass transfer. Experimental results of the correlations between $D_{\mathrm{e}}$ and $\varepsilon_{\mathrm{e}}$ and that between $D_{\mathrm{s}}$ and $\varepsilon_{\mathrm{e}}$ are illustrated. This material is available free of charge on the Web at http://www.jsac.or.jp/analsci/.

\section{References}

1. G. Guiochon, J. Chromatogr. A, 2007, 1168, 101.

2. V. González-Ruiz, A. I. Olives, and M. A. Martín, $\operatorname{Tr} A C$,
Trends Anal. Chem., 2015, 64, 17.

3. J. C. Giddings, "Dynamics of Chromatography", 1965, Marcel Dekker, New York.

4. J. J. van Deemter, F. J. Zuiderweg, and A. Klinkenberg, Chem. Eng. Sci., 1956, 5, 271.

5. K. Miyabe and G. Guiochon, Adv. Chromatogr., 2000, 40, 1.

6. K. Miyabe and G. Guiochon, J. Chromatogr. A, 2010, 1217, 1713.

7. D. M. Ruthven, "Principles of Adsorption \& Adsorption Processes", 1984, John Wiley and Sons, New York.

8. M. Suzuki, "Adsorption Engineering", 1990, Kodansha/ Elsevier, Tokyo/Amsterdam.

9. G. Guiochon, S. Golshan-Shirazi, and A M. Katti, "Fundamentals of Preparative and Nonlinear Chromatography", 1994, Academic Press, Boston.

10. S. Fanali, P. R. Haddad, C. F. Poole, P. Schoenmakers, and D. Lloyd, "Liquid Chromatography: Fundamentals and Instrumentation", 2013, Elsevier, Amsterdam.

11. K. Miyabe and G. Guiochon, J. Phys. Chem. B, 2002, 106, 8898.

12. K. Miyabe, Anal. Chem., 2007, 79, 7457.

13. K. Kaczmarski and G. Guiochon, Anal. Chem., 2007, 79, 4648.

14. K. Miyabe, J. Chromatogr. A, 2008, 1183, 49.

15. K. Miyabe, Anal. Sci., 2011, 27, 1007.

16. K. Miyabe and G. Guiochon, J. Sep. Sci., 2003, 26, 155.

17. K. Miyabe and G. Guiochon, J. Sep. Sci., 2004, 27, 853.

18. K. Miyabe, J. Sep. Sci., 2009, 32, 757.

19. K. Miyabe, TrAC, Trends Anal. Chem., 2016, 81, 79.

20. K. Miyabe and G. Guiochon, Anal. Chem., 2011, 83, 182.

21. H. Kobayashi, D. Tokuda, J. Ichimaru, T. Ikegami, K. Miyabe, and N. Tanaka, J. Chromatogr. A, 2006, 1109, 2.

22. C. R. Wilke and P. Chang, AIChE J., 1955, 1, 264.

23. B. E. Poling, J. M. Prausnitz, and J. P. O'connell, "The Properties of Gases and Liquids", 2001, McGraw-Hill, New York.

24. M. J. M. Wells and C. R. Clark, Anal. Chem., 1981, 53, 1341.

25. M. Al-Bokari, D. Cherrak, and G. Guiochon, J. Chromatogr. A, 2002, 975, 275.

26. D. Cabooter, F. Lynen, P. Sandra, and G. Desmet, $J$. Chromatogr. A, 2007, 1157, 131.

27. J. Billen and G. Desmet, J. Chromatogr. A, 2007, 1168, 73.

28. J. S. Baker, J. C. Vinci, A. D. Moore, and L. A. Colón, J. Sep. Sci., 2010, 33, 2547.

29. F. Gritti and G. Guiochon, J. Chromatogr. A, 2012, 1221, 2.

30. K. Miyabe, Y. Sakai, M. Sarashina, and C. Yokokawa, Analyst, 2003, 128, 1425.

31. K. Miyabe, H. Kobayashi, D. Tokuda, and N. Tanaka, J. Sep. Sci., 2006, 29, 2452.

32. K. Miyabe, Y. Matsumoto, and G. Guiochon, Anal. Chem., 2007, 79, 1970.

33. K. Miyabe, A. Cavazzini, F. Gritti, M. Kele, and G. Guiochon, Anal. Chem., 2003, 75, 6975.

34. N. Tanaka, H. Kobayashi, K. Nakanishi, H. Minakuchi, and N. Ishizuka, Anal. Chem., 2001, 73, 420A.

35. F. Svec, T. B. Tennikova, and Z. Deyl, "Monolithic Materials-Preparation, Properties and Applications, Journal of Chromatography Library, 67', 2003, Elsevier, Amsterdam.

36. D. Hlushkou, S. Bruns, A. Höltzel, and U. Tallarek, Anal. Chem., 2010, 82, 7150. 